\title{
Do pre-operative blood tests predict patient length of stay in elective total hip replacement?
}

Background: Enhanced recovery begins before the onset of surgery with a thorough pre-operative assessment prior to their procedure. Numerous benefits have been proven from its implementation. Reducing postoperative complications, saving costs, shortening length of stay (LOS), improving patient satisfaction, and promoting faster recovery, are to name a few. Part of the pre-operative assessment includes blood investigations. This study aims to determine whether preoperative blood tests, normal or abnormal, have an effect on a patient's post-operative length of stay.

Objectives: To determine whether pre-operative blood tests, namely haemoglobin ( $\mathrm{Hb}$ ) levels, white cell count (WCC) and renal function tests (with a focus on the estimated glomerular filtration rate (eGFR)), have an effect on patient's total length of stay in hospital.

Study design \& methods: A retrospective analysis of pre-operative blood tests, Hb levels, WCC and the eGFR, compared to the total length of stay in hospital, post elective total hip replacement (THR). Over a 9-month period (from January to September 2017), we analyzed 51 patient records who underwent elective total hip arthroplasty within our unit.3 variables were looked at, and these included: $\mathrm{Hb}$ levels during admission (prior to surgery), WCC during admission and renal function during admission. Each were compared to the patient's length of stay. Confounding factors were removed from the study, which largely consisted of the social issues delaying a patient's discharge. Our data takes into account patients who received a blood transfusion, patients who required antibiotics for a post-operative infection and those patients who required Intensive care (ITU) or High Dependency Unit (HDU) level care.

Results: A total of 51 patients were identified, who underwent total hip replacement during this time period. Our data concluded that of these 51 patients, 21 (41\%) were found to have a pre-operative $\mathrm{Hb}$ below $109 \mathrm{~g} / \mathrm{L}$ and of them $13(61 \%)$ were subsequently discharged later than scheduled (beyond 7 days). Five of these 13 patients (38\%) required a postoperative blood transfusion.

Of the remaining 30 patients who had a pre-operative $\mathrm{Hb}$ greater than $109 \mathrm{~g} / \mathrm{L}, 22$ (73\%) were discharged early (within 4 days), the remaining 8 patients (26\%) were discharged from hospital within 1 week.

We also found that of the 51 patients, 17 (33\%) patients had a raised pre-operative WCC, of these 17, 9 (53\%) were discharged more than 7 days after their surgery. From these 17 patients who had a raised pre-operative WCC and were discharged late, 5 (29\%) were treated for a post-operative urinary tract infection (UTI), and 3 patients (18\%) were treated for a hospital acquired pneumonia.

Of the 51 patients; only 18 (35\%) had an eGFR >90, 20 patients (39\%) had an eGFR between 60-90, and 13 patients (25\%) had an eGFR $<60$. Of those patients with an eGFR $<60,10$ were discharged more than 4 days after their surgery. Six of these 10 patients $(60 \%)$ were discharged more than 7 days post-surgery.

Conclusion: From the data collected, it is apparent that preoperative investigations prior to and during admission do have an almost predictable effect on the length of stay, and improving these variables would improve the post-operative outcome for our patients. It is imperative that these pre-operative investigations are rigorously analysed prior to the patient being listed for surgery.t.

Keywords: total hip replacement, estimated glomerular filtration rate, intensive care unit, high dependency unit, urinary tract infection, white cell count, orthopaedic surgery, elective surgery, enhanced recovery, length of stay.

\section{Introduction}

From the point at which a patient is seen in the outpatient clinic, we can risk stratify those patients as high or low risk, prior to listing them for surgery. This initial inkling is then quantified further by means of pre-operative investigations. Elective total hip replacement surgery in itself is generally regarded as a high risk procedure, 
given that in most cases operative time is more than 1 hour and patients who require this procedure are elderly and do have other co-morbidities. Hence, pre-operative blood tests for elective total hip replacement are now common practice. In addition to evaluating the patients baseline prior to their surgery, pre-operative blood tests can be used to help identify problems, both potential and real, and subsequently delay a procedure until the patient has been appropriately optimised.

**Total hip replacement facilitates early mobilisation and early discharge of these patients, with some trusts now offering elective THR as day case surgery. Extended LOS is unfavorable and has been shown to be associated with an increased risk of post-operative infections, worsened patient outcomes and high levels of dissatisfaction.

Other authors have looked into patient factors which help to predict post-operative outcome following orthopaedic surgery, and have focused primarily on age, gender, socioeconomic status, pre-operative function, pain and quality of life [1]. Other factors such as pre-operative blood tests were not looked into thoroughly, and have been under-reported.

The laboratory tests focused on in this study are the haemoglobin levels, total white cell count and eGFR. This study attempts to explore whether the results of routine pre-operative laboratory testing affect the post-operative outcomes, and hospital length of stay.

Anaemia has been found to correlate with a high risk of complications during the perioperative period [2]. Transfusion is an effective treatment for pre and post-operative anaemia, and it was thought that transfusion may reduce the rate of anemia-related post-operative complications. However, transfusion itself can have adverse effects including pulmonary complications, graft versus host disease, and the transmission of infectious diseases. Several reports have suggested that the transfusion, and not anemia, increases the risk of post-operative complications, which directly prolongs the hospital length of stay [3].

On the other hand, leukocytosis is a nonspecific marker for a systemic inflammatory state [4]. Surgical trauma induced nonspecific inflammation is possibly the "second hit" in patients with a pre-existing systemic inflammatory state, plausibly associated with adverse outcome [5]. Systemicinflammation after major surgeries is a well-known phenomenon that is attributable to various mechanisms. This vigorous non-specific inflammatory state with clinical sequelae is referred to as the systemic inflammatory response syndrome (SIRS) [6]. Leukocytosis is amongst the criteria used for diagnosing SIRS [7]. Multiple focused studies have demonstrated the value of leukocytosis as a marker of specific outcomes such as morbidity and mortality, however, not many studies focused on post-operative hip related surgical outcomes. These studies did not exclude known causes of leukocytosis which limited their ability to conclude a direct relationship between leukocytosis and adverse post-operative outcomes. As such, an elevated pre-operative WBC count was not considered a risk factor, something we hoped to address in this study.

In addition to leukocytosis, acute renal dysfunction is an established marker of an adverse outcome, in particular cardiovascular complications, in many settings [8]. Its role in patients undergoing surgery, however, is not as clearly defined. A variety of more precise measures have been described and used for risk stratification, in particular, the estimated glomerular filtration rate (eGFR), which is a more accurate indicator of renal function [9], identifies milder degrees of renal dysfunction [10], and predicts cardiovascular and renal outcomes in diverse populations [11].

\section{Materials and Methods}

\section{- Study design}

Patients undergoing total hip replacement were included in a retrospective observational study looking directly into their haemoglobin levels, total white cell count and eGFR preoperatively. These haematological levels were then assessed and compared against their postoperative inpatient length of stay and other associated complications.

\section{- Patients and settings}

A total of 51 patients undergoing total hip replacement (THR) at Good Hope Hospital were included in the study between January 2017 and September 2017. Data was collected from intranet theatre lists, hospital laboratory results, patient's notes and discharge letters.

\section{A Saad ${ }^{1 *}$, S Shahban ${ }^{1 *}$, P Sonsale ${ }^{2}$, R Fernandes ${ }^{2}$}

${ }^{1}$ Birmingham Heartlands Hospital, Birmingham, United Kingdom ${ }^{2}$ Good hope Hospital, Birmingham, United Kingdom

*Author for correspondence:

aisaad@hotmail.co.uk; shafiqshahban@hotmail.com 
Exclusion criteria included any patients that had social issues delaying their discharge. These comprised of patients awaiting package of care placements, further non-acute NHS care or patient/family choice.

All patients who had adverse post-operative outcomes, including any morbidity or mortality that had a frank effect on hospital length of stay were included in the study.

\section{- Variables}

The notes and patient details of our cohort of 51 patients were reviewed and 3 variables were analysed and compared against the hospital LOS. For similar patients undergoing hip surgery in other institutions, we found that the average length of stay in hospital was around 7 days [12]. Of the patients in our group we stratified LOS into 3 categories; early discharge within 3 days, late discharge beyond 7 days and those patients in between (between 4-7 days).

Of the haemoglobin levels that we assessed, we labelled a haemoglobin of less that $109 \mathrm{~g} / \mathrm{l}$ as being anaemic, above $125 \mathrm{~g} / \mathrm{l}$ as being normal and between $110-124 \mathrm{~g} / \mathrm{l}$ as being low. This was in line with the hospital pathology lab criteria.

A normal white cell count (WCC) was recorded between 4000-11000 cells/L. Levels above or below this were abnormal. Again this was in keeping with our hospital pathology protocol.

Renal function was assessed by looking at the eGFR. These rates were then broken down further into 3 categories; normal (above $90 \mathrm{~mL} /$ $\left.\mathrm{min} / 1.73 \mathrm{~m}^{2}\right)$, low $\left(<60 \mathrm{~mL} / \mathrm{min} / 1.73 \mathrm{~m}^{2}\right)$ and in between $\left(60-90 \mathrm{~mL} / \mathrm{min} / 1.73 \mathrm{~m}^{2}\right)$.

\section{Results}

As a group, out of a total of 51 patients, 23 (41.8\%) were discharged early (within 4 days), 18 patients (32.7\%) were discharged late (8 days or more post-surgery), and the remaining $14(25.5 \%)$ being discharged in between these 2 groups (between 5-7 days). TABLE 1 demonstrates these results.

\section{- Hb levels vs LOS}

Thirty out of the 51 patients $(58.8 \%)$ had a pre-operative haemoglobin level higher than 109 $\mathrm{g} / \mathrm{L}$. Of these, 17 (56.7\%) patients had normal haemoglobin levels (above $125 \mathrm{~g} / \mathrm{l}$ ). We then correlated these results against LOS and found that 29 patients with a $\mathrm{Hb}$ of more than 109 $\mathrm{g} / \mathrm{l}$ were discharged within 1 week. Whereas the 13 patients with a $\mathrm{Hb}$ of less than $109 \mathrm{~g} / \mathrm{l}$ were discharged late (7 days' post-surgery). TABLE 2 and FIGURE 1 demonstrates these findings.

\section{WCC vs LOS}

We found that out of the 51 patients undergoing hip related surgery, 34 (66.7\%) had a pre-operative white cell count (WCC) within normal limits, and 18 patients $(35.3 \%)$ had a raised WCC (greater than 11,000 cells/l).

Of the 25 patients that were discharged early (within 4 days), 22 (88\%) had normal WCC. The remaining 3 patients (12\%) had asymptomatic leukocytosis.

Fourteen patients were discharged late, and out of these $9(64.3 \%)$ had a raised pre-operative WCC. Of them, 5 (35.7\%) were treated for a post-operative urinary tract infection (UTI), 3 patients $(21.4 \%)$ were treated for a hospital acquired pneumonia (HAP). 2 were treated for post-operative wound infections, bacillus was the organism isolated in both cases, and 1 patient suffered a myocardial infarction. There was no clear documentation for the remaining 3 patients. TABLE 3 and FIGURE 2 demonstrate these findings.

\section{eGFRvs LOS}

When comparing the eGFR to LOS in the group of patients who were discharged early (within 4 days' post-surgery), we found that less than half of all the patients had an eGFR level $>90 \mathrm{~mL} / \mathrm{min} / 1.73 \mathrm{~m}^{2}(12 / 18,67 \%)$. The remaining 6 patients $(33 \%)$ had an eGFR $<90$ $\mathrm{mL} / \mathrm{min} / 1.73 \mathrm{~m}^{2}$.

Ten patients with a pre-operative eGFR of $<60 \mathrm{~mL} / \mathrm{min} / 1.73 \mathrm{~m}^{2}$ were discharged more than 4 days after their surgery, with the majority of this group actually being discharged late (more than a week post-surgery). TABLE 4 and FIGURE 3 highlight these results.

\begin{tabular}{|c|c|}
\hline $\begin{array}{l}\text { Day of discharge } \\
\text { (post-surgery) }\end{array}$ & No. of patients \\
\hline 1-4 (Early) & $25(49 \%)$ \\
\hline 5-7 (Middle) & 12 (23.5\%) \\
\hline$>7$ (Late) & $14(27.4 \%)$ \\
\hline Total & 51 \\
\hline
\end{tabular}


Table 2. Comparison of the pre-operative Hb levels against LOS.

Early (0-4 days) Middle (5-7 days) Late ( $>7$ days) TOTAL

\begin{tabular}{|c|c|c|c|c|}
\hline $125-160 \mathrm{~g} / \mathrm{l}$ & 15 (88\%) & $1(6 \%)$ & $1(6 \%)$ & 17 \\
\hline $109-124 \mathrm{~g} / \mathrm{l}$ & 7 (54\%) & $6(46 \%)$ & $0(0 \%)$ & 13 \\
\hline$<109 q / /$ & $3(14 \%)$ & $5(24 \%)$ & $13(62 \%)$ & 21 \\
\hline
\end{tabular}

$\mathrm{Hb}$ levels Vs Length Of Stay

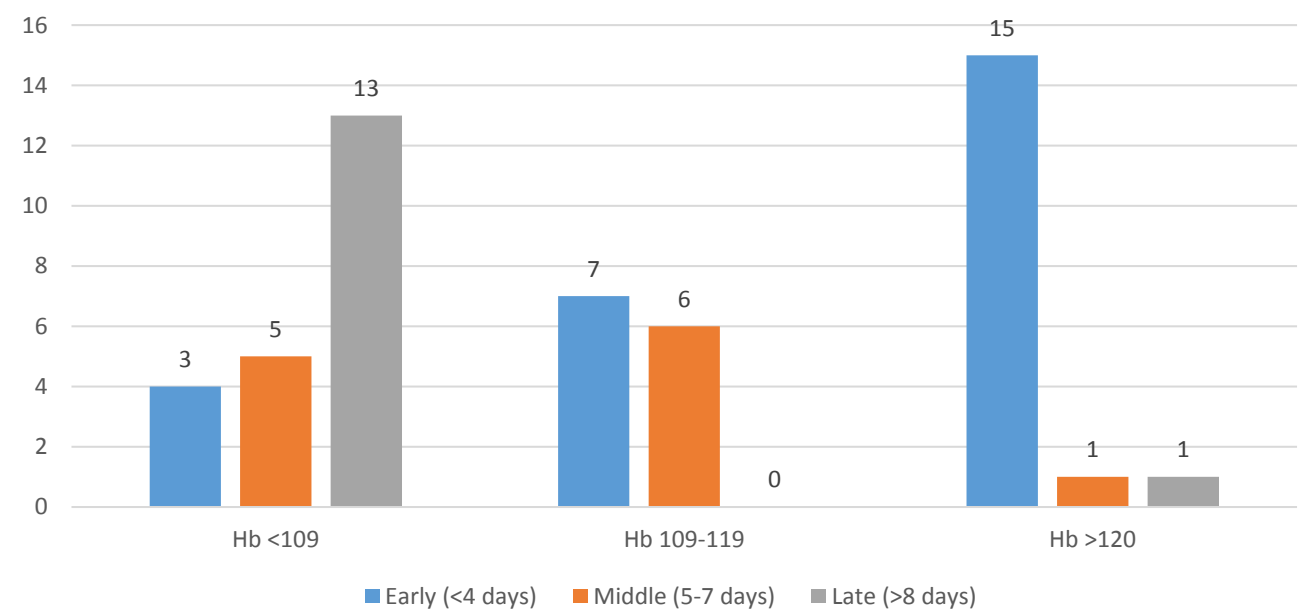

FIGURE 1. Graphical demonstration of the pre-operative Hb against LOS

\begin{tabular}{|c|c|c|c|c|}
\hline WCC & Early (<4 days) & Middle (5-7 days) & Late (>8 days) & WCC \\
\hline 4-11 & $22(65 \%)$ & $7(21 \%)$ & $5(15 \%)$ & 4-11 \\
\hline$>11$ & $3(18 \%)$ & $5(29 \%)$ & $9(53 \%)$ & $>11$ \\
\hline
\end{tabular}

WCC Vs Length Of Stay

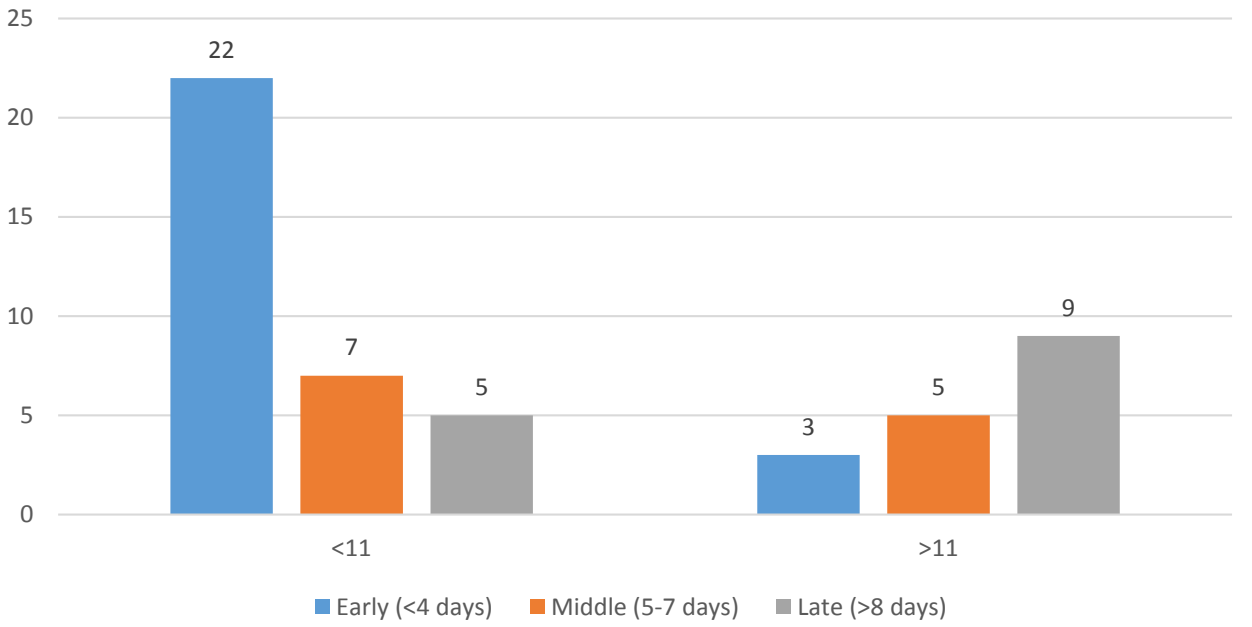

FIGURE 2. Graph comparing pre-operative WCC level and LOS.

\begin{tabular}{|c|c|c|c|c|}
\hline \multicolumn{2}{|c|}{ Table 4.Comparison of pre-operative eGFRvs LOS. } \\
\hline eGFR & Early (<4 days) & Middle (5-7 days) & Late (>8 days) & TOTAL \\
\hline $\mathbf{> 9 0}$ & $12(67 \%)$ & $4(22 \%)$ & $2(11 \%)$ & 18 \\
\hline $\mathbf{6 0 - 9 0}$ & $10(50 \%)$ & $4(20 \%)$ & $6(30 \%)$ & 20 \\
\hline$<\mathbf{6 0}$ & $3(23 \%)$ & $4(31 \%)$ & $6(46 \%)$ & 13 \\
\hline
\end{tabular}




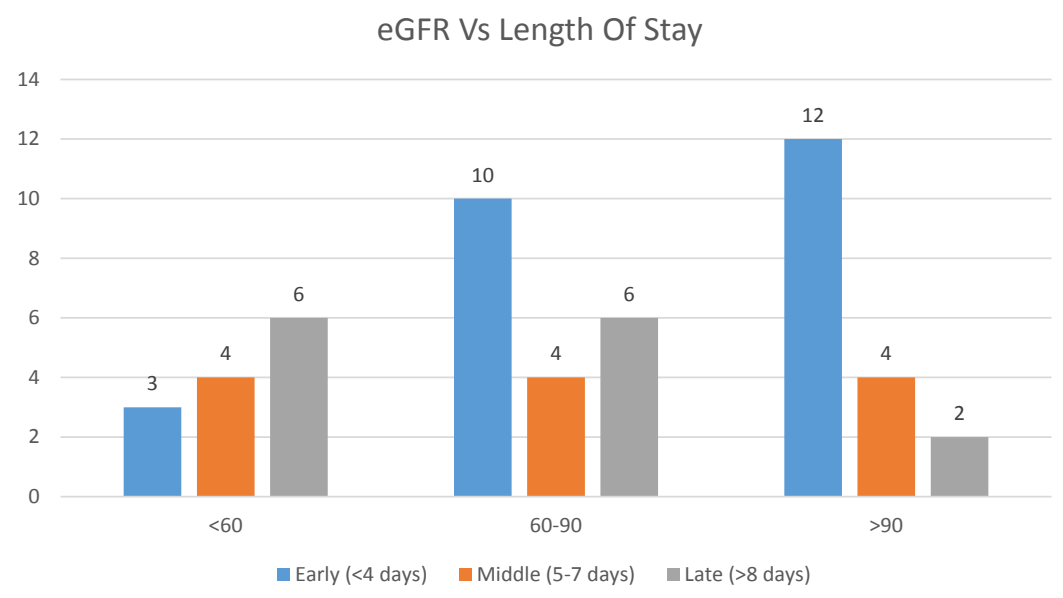

FIGURE 3. Graphical representation of the pre-operative eGFRvs LOS

\section{Discussion}

The purpose of this study was to evaluate the association of pre-operative blood tests, focusing mainly on the haemoglobin levels, total white cell count and eGFR, with post-operative hospital length of stay and post-operative complications.

Some studies have demonstratedhow pre-operative anaemia increases the rate of post-operative complications [13], thereby, prolonging hospital LOS. Similar studies investigating this have found how a mild anaemia does not affect the rate of post-operative complications [14].

From our cohort of patients undergoing elective THR, we can see the association of pre-operative anaemia and prolonged hospital LOS. From this group of 22 patients, we also saw that $5(22.7 \%)$ required a postoperative blood transfusion, and of course receiving a blood transfusion in itself can be a risk factor for developing other post-transfusion complications $[15,16]$. Equally, we found that all 5 of these patients who required a blood transfusion had prolonged hospital LOSs of more than a week post-surgery. Usually blood transfusion is not mandatory in patients with $\mathrm{Hb} \geq 9 \mathrm{~g} / \mathrm{dL}$ [17]; however, we found that the indication(s) for a post-operative blood transfusion did vary greatly.

Normalisation of a pre-operative haemoglobin, we feel, is something that should commence within the community, by the patient's general practitioner. In doing this, not only can we reduce the risk of requiring a blood transfusion post-operatively, but we can also reduce their hospital length of stay as well as reducing their risk of developing any other post-operative complications.

Our study has also demonstrated that leukocytosis, symptomatic or asymptomatic, is an independent predictor of morbidity and thus prolonged length of stay post-operatively. The association between asymptomatic leukocytosis and the composite outcome of medical complications is of particular importance. These findings may point towards the possibility of an exaggerated subclinical inflammatory response. Therefore, pre-operative asymptomatic leukocytosis may warrant an additional level of scrutiny to investigate and rectify this prior to surgery. Our results highlight, that in doing this we reduce length of stay and the risk of developing any other post-operative complications.

Several recent studies have also shown an association between pre-operative leukocytosis and post-operative complications across a wide variety of other surgeries. One study in particular was undertaken to determine if preoperative fever and leukocytosis without an established source of untreated infection are independent risk factors for the development of deep post-operative wound infection (DPWI) post-surgical fixation of pelvic and acetabular fractures. This study found that 17 patients $(2.8 \%)$ with a pre-operative fever or leukocytosis developed DPWI, distributed as $8(2.3 \%)$ acetabular, 5 (2.9\%) pelvic, and 4 (5.4\%) pelvicacetabular infections [18]. Similarly, within cardiothoracics, post-operative pneumonia is 
the most common infection following cardiac surgery, and is a significant source of morbidity and mortality [19]. In one study looking at the clinical outcomes of elderly hip fracture patients who underwent surgery compared to those who did not, the three most common complications were urinary tract infection (1.9\%), pressure sores $(1.0 \%)$ and wound infection (0.7\%) [20].

From our study, we saw that $21.4 \%$ of patients with a raised pre-operative WCC went on to develop hospital acquired pneumonia post-operatively and a significant proportion of these $(35.7 \%)$ developed symptomatic UTI, warrantingtreatment with intravenous (IV) antibiotics and subsequently delaying their discharge.

Incorporating WCC count as a risk marker can have significant clinical implications. While there is value in incorporating leukocytosis into surgical risk assessment models in order to improve risk assessment, the major advantage of this information may potentially lie in mitigating the inflammatory response [21]. Clinicians should adequately assess patients during admission, and a raised WCC should warrant further investigations to look for a potential source, with further management instigated as appropriate.

Finally, this study illustrates that the patients with a pre-operative eGFR level below $60 \mathrm{~mL} /$ $\mathrm{min} / 1.73 \mathrm{~m}^{2}$ were all discharged late. In patients undergoing surgery, chronic kidney disease is recognised as an important determinant of a poor prognosis and is included in several, though not all, risk scores, [22]. The eGFR is now the recognised measure of choice for renal function and has in recent years been increasingly used in the pre-operative setting. In particular, some studies have shown how eGFR can predict both short- ( $<30$ days) and long-term mortality postsurgery.

Of the 13 patients found to have a low eGFR $\left(<90 \mathrm{~mL} / \mathrm{min} / 1.73 \mathrm{~m}^{2}\right)$, only 7 were known to have chronic kidney disease (CKD), and subsequently only 4 out of these 7 were discharged within a week. Pre-operative interventions such as pre-operative hydration and limitation of the use of nephrotoxins is paramount in this patient group, and something which needs to be highlighted. Not only for the patients with known CKD, but those without this diagnosis with an eGFR of $<60 \mathrm{~mL} /$ $\min / 1.73 \mathrm{~m}^{2}$ requires at least a discussion with a nephrologist, prior to referral for consideration of surgery. Equally abnormalities such as this need identifying and acting upon within the pre-operative assessment setting.

\section{Conclusion}

In conclusion, our retrospective analysis of our patients at Good Hope Hospital undergoing elective THR suggests that a pre-operative low $\mathrm{Hb}$, elevated WBC count and low eGFR, are all strong predictors of increased post-operative length of stay, and potentially the development of post-operative complications. Based on these findings we strongly encourage all clinicians involved in the surgery of these patients to be aware of the pre-operative blood tests, and for those patients who do fall out of the "normal" haematological ranges, then strongly consider delaying surgery until it is safe to proceed. Equally, all patients who do have abnormal blood tests who do undergo elective hip surgery, require close monitoring for the potential of developing post-operative complications in addition to acknowledging that these patients may well have delayed discharges. 


\section{REFERENCES}

Waheeb A, Zywiel MG, Palaganas M, Venkataramanan V, Davis AM. The influence of patient factors on patientreported outcomes of orthopedic surgery involving implantable devices: a systematic review. Semin. Arthritis.Rheum. 44(4), 461-471 (2015).

Leichtle SW, Mouawad NJ, Lampman R, Singal B, Cleary RK. Does preoperative anemia adversely affect colon and rectal surgery outcomes? J. Am. Coll. Surg. 212(2), 187-194 (2011).

Yuruk K, Bartels SA, Milstein $\mathrm{DM}$, Bezemer R, Biemond BJ, Ince C. Red blood cell transfusions and tissue oxygenation in anemichematology outpatients. Transfusion 52(3), 641-646 (2012).

Bennis M, Parc Y, et al. Morbidity risk factors after low anterior resection with total mesorectal excision and coloanal anastomosis: a retrospective series of 483 patients. Ann. Surg. 255(3), 504-10 (2012).

MG Davies, PO Hagen. Systemic inflammatory response syndrome. $B r . J$. Surg. 84(7), 920-935 (1997).

Paparella D, Yau TM, Young E. Cardiopulmonary bypass induced inflammation: pathophysiology and treatment. An update. Eur. J. Cardiothorac Surg. 21(2), 232-244 (2002).

Lindner HA, Balaban Ü, Sturm T, Weiß C, Thiel M, Schneider-Lindner V. 2016. An Algorithm for Systemic Inflammatory Response Syndrome Criteria-Based Prediction of Sepsis in a Polytrauma Cohort. Crit. Care Med. 44(12),2199-2207 (2016).

Tonelli M, Wiebe N, Culleton B, et al. Chronic kidney disease and mortality risk: a systematic review. J. Am. Soc. Nephrol. 17(7), 2034-2047 (2006).

National Kidney Foundation. K/ DOQI clinical practice guidelines for chronic kidney disease: Evaluation, classification, and stratification. Am. J. Kidney Dis. 39(2S1), S1-266

Go AS, Chertow GM, Fan D, McCulloch CE, et al. Chronic kidney disease and the risks of death, cardiovascular events, and hospitalization. N. Engl. J. Med. 351,1296-1305 (2004).

Gibson PH, Croal BL, Cuthbertson $\mathrm{BH}$, et al.The relationship between renal function and outcome from heart valve surgery.Am. Heart J.156, 893-912 (2008).

Bernard AC, Davenport DL, Chang $\mathrm{PK}$, et al. Intraoperative transfusion of $1 \mathrm{U}$ to $2 \mathrm{U}$ packed red blood cells is associated with increased 30-day mortality, surgicalsite infection, pneumonia, and sepsis in general surgery patients. J. Am. Coll. Surg. 208(5), 931-937 (2009).

Napolitano LM, Kurek S, Luchette FA, et al. Clinical practice guideline: red blood cell transfusion in adult trauma and critical care. Crit. Care Med. 67(6),14391442 (2009).

Jung DH, Lee HJ, Han DS, et al. Impact of perioperative hemoglobin levels on postoperative outcomes in gastric cancer surgery. Gastric Cancer. 16(3),377382 (2013).
Sagi HC, Dziadosz D, Mir H, Virani $\mathrm{N}$, Olson C. Obesity, leukocytosis, embolization, and injury severity increase the risk for deep postoperative wound infection after pelvic and acetabular surgery. J. Orthop. Trauma. 27(1),6-10 (2013).

Kollef MH, Sharpless L, Vlasnik J, et al. The impact of nosocomial infections on patient outcomes following cardiac surgery. Chest. 112(3), 666-675 (1997).

Tan STS, Tan WPM, Jaipaul J, Chan SP, Sathappan SS. Clinical outcomes and hospital length of stay in 2,756 elderly patients with hip fractures: a comparison of surgical and non-surgical management. Singapore Med. J. 58(5),253-257 (2017).

Mahmood E, Knio ZO, Mahmood $\mathrm{F}$, et al. Preoperative asymptomatic leukocytosis and postoperative outcome in cardiac surgery patients. PLoS One. 12(9), e0182118 (2017).

Nashef SA, Roques F, Michel Pal, et al. European system for cardiac operative risk evaluation (EuroSCORE). Eur. J. Cardiothorac. Surg. 16(1),9-13(1999).

Goldman L, Caldera DL, Nussbaum SR. Multifactorial index of cardiac risk in noncardiac surgical procedures. N. Engl. J. Med. 297(3), 845-850 (1977).

Mooney JF, Ranasinghe I, Chow CK. Preoperative estimates of glomerular filtration rate as predictors of outcome after surgery: a systematic review and meta-analysis. Anesthesiology. 118(4),809824 (2013). 